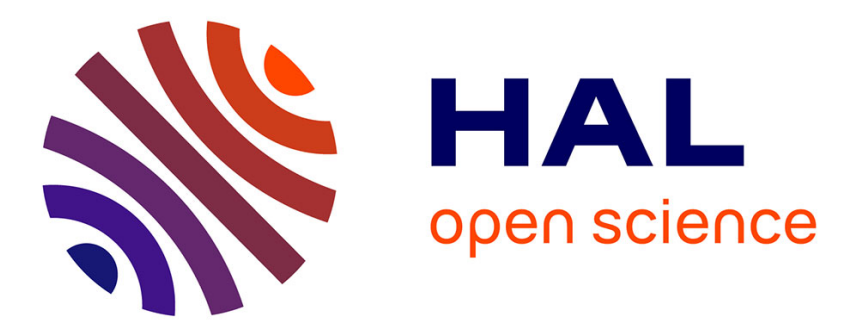

\title{
Stabilization of the planar vertical take-off and landing using nonlinear feedback control
}

\author{
Rogelio Lozano, Sergio Salazar, Jonathan Flores
}

\section{To cite this version:}

Rogelio Lozano, Sergio Salazar, Jonathan Flores. Stabilization of the planar vertical take-off and landing using nonlinear feedback control. International Journal of Robust and Nonlinear Control, 2022, 32, pp.3-12. 10.1002/rnc.5808 . hal-03411722

\section{HAL Id: hal-03411722 \\ https://hal.science/hal-03411722}

Submitted on 2 Nov 2021

HAL is a multi-disciplinary open access archive for the deposit and dissemination of scientific research documents, whether they are published or not. The documents may come from teaching and research institutions in France or abroad, or from public or private research centers.
L'archive ouverte pluridisciplinaire HAL, est destinée au dépôt et à la diffusion de documents scientifiques de niveau recherche, publiés ou non, émanant des établissements d'enseignement et de recherche français ou étrangers, des laboratoires publics ou privés. 


\title{
Stabilization of the PVTOL using nonlinear feedback control
}

\author{
Rogelio Lozano ${ }^{1}$, Sergio Salazar ${ }^{2}$, and Jonathan Flores ${ }^{2}$ \\ ${ }^{1}$ UMR 7253 Heudiasyc, Sorbonne Universités, UTC, CNRS, \\ Compiègne, France. \\ 2UMI-LAFMIA 3175 CNRS, CINVESTAV-IPN, Mexico City, \\ Mexico.
}

\begin{abstract}
This paper presents a new control strategy for the well known problem of the PVTOL. The total thrust is computed using a non linear feedback compensation so that the altitude reaches the desired altitude. The horizontal position $x$ is then controlled by choosing the orientation angle $\theta$ as a smooth saturation function of $x$ and $\dot{x}$. A proof of convergence is presented using a Lyapunov approach. The proposed control strategy is successfully tested in numerical simulations.
\end{abstract}

Keywords: PVTOL, nonlinear systems, saturation functions, helicopter control

\section{Introduction}

The PVTOL (Planar Vertical Take-Off and Landing) problem has attracted a lot of attention during the last decades. The reason for such an interest stems from the fact that it is an important theoretical problem in the automatic control community which is clearly related to a practical application. Indeed, the PVTOL is a nonlinear dynamical system that represents the simplified longitudinal model of a helicopter or a quadrotor. The dynamic model is very simple but contains two trigonometric functions which make the stabilization problem quite challenging.

One of the first papers to deal with the stabilization of the PVTOL was (1). This paper motivated the search for control algorithms to stabilize the PVTOL. Since the publication of (1), different techniques have been proposed to control the PVTOL. Optimal controllers were presented in $(2-5)$ to stabilize the PVTOL. Nonlinear feedback linearization techniques to control the PVTOL have been developed in (6-13). The sliding mode controller design has also been applied to the PVTOL in (14-17). Furthermore, the backstepping approach has been used in (18-21). Other well known techniques have been proposed to control the PVTOL as prediction based control (22), gain scheduling (23), singular perturbation (24), fault tolerant control (25), Lyapunov approach (26, 27), quaternions technique (28) and adaptive control (29, 30).

(31) and (32) proposed the use of a nested saturation control algorithm (33) to stabilize the PVTOL.

An interesting control algorithm for the PVTOL was proposed in (34). They used the concept of virtual or ideal forces such that the PVTOL model becomes fully actuated. 
The orientation angle and the thrust required to generate such virtual forces are then computed. The first and second order derivatives of the orientation are used in the control algorithm and therefore the virtual forces have to be smooth functions.

A controller was presented in (35) which is based on a combination of the traditionally PD-controller and a sliding mode controller to stabilize both the horizontal and angular variables to the desired rest position. A hierarchical control strategy was proposed in (36) for a miniature VTOL vehicle to track a desired trajectory globally with respect to the initial position and attitude configuration.

The contribution of paper is as follows. The PVTOL has three degree of freedom which are the displacement $x$, the altitude $z$ and the angular displacement $\theta$. The total thrust $u$ is currently used to stabilize the altitude $z$ which converges to a desired value. The system is then essentially reduced to the form $\ddot{x}=\tan (\theta)=u_{1}$ and $\ddot{\theta}=\tau$. The problem is to find an expression for $\tau$ such that $x$ converges to a desired value. One way to solve the problem is to assign a virtual input $u_{1}$ as a function of $x$ and $\dot{x}$, to stabilize $x$. The desired value for $\theta$ will then be $\theta_{d}=\arctan \left(u_{1}\right)$. In order to obtain a control input $\tau$ so that $\theta$ converges to $\theta_{d}$ we will require the first and second derivatives of $\theta_{d}$. This technique has been used in (34). In the present paper we have been able to simplify the expression for $\theta_{d}$ to only $\theta_{d}=u_{1}$ without the arctan function. The computation of the first and second derivatives of $\theta_{d}$ are much simpler since arctan does no longer appear in $\theta_{d}$. Furthermore, the contribution can also be presented in the following conceptual alternative way. Consider the first order non linear system $\dot{x}=\tan (\theta)$. We can use the fact that $\tan (\theta)$ and $\theta$ have the same sign and propose the simple desired angle $\theta_{d}=-x$ which does not include arctan. When $\theta=\theta_{d}$ we get $\dot{x}=-\tan (x)$ which is Lyapunov stable. The question is whether the above technique can be extended to the second order nonlinear system $\ddot{x}=\tan (\theta)$ without recurring to the arctan function. The present paper presents a solution to the above problem and applies it to the stabilization of the PVTOL.

The paper is organized as follows : Section ?? presents the PVTOL model. Section 3 describes the altitude control law. Section 4 is devoted to the controller for the horizontal displacement $x$. The orientation control algorithm is given in Section 5 The numerical simulations of the proposed control strategy are shown in Section 6 . Final remarks are given in the conclusions.

\section{PVTOL mathematical model}

The PVTOL model can be written as

$$
\begin{aligned}
m \ddot{x} & =u \sin \theta \\
m \ddot{z} & =u \cos \theta-m g \\
\ddot{\theta} & =\tau
\end{aligned}
$$

Figure 1 presents the PVTOL where $f_{1}$ and $f_{2}$ are the control forces, $m$ is the mass, $\theta$ is the angle of the aircraft with respect to the horizontal line, $g$ is the gravitational acceleration, $x$ is the horizontal displacement and $z$ is the vertical displacement. Let us define the total thrust $u$ and the torque $\tau$ respectively as follows

$$
\begin{aligned}
& u=f_{1}+f_{2} \\
& \tau=\left(f_{1}-f_{2}\right) l
\end{aligned}
$$




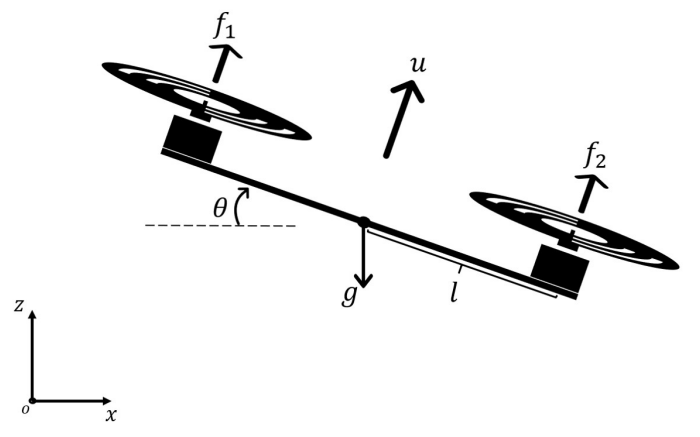

Figure 1: The PVTOL diagram.

\section{Altitude control $(z)$}

From (1) it follows that

$$
\begin{gathered}
m \ddot{x}=u \sin \theta_{d}+\epsilon_{1} \\
m \ddot{z}=u \cos \theta_{d}-m g+\epsilon_{2}
\end{gathered}
$$

where

$$
\begin{aligned}
& \epsilon_{1}=u\left(\sin \theta-\sin \theta_{d}\right) \\
& \epsilon_{2}=u\left(\cos \theta-\cos \theta_{d}\right)
\end{aligned}
$$

where $\theta_{d}$ is the desired orientation. The control input $\tau$ will be computed so that $\theta-\theta_{d}$ converges to zero exponentially

In order to control the vertical displacement $z$ let us choose the following control input $u$ where $z_{d}$ is the constant desired altitude.

$$
u=\frac{m}{\cos \theta_{d}}\left[g-2 \dot{z}-\left(z-z_{d}\right)\right]
$$

To avoid singularities in the above equation the desired angular position $\theta_{d}$ will be chosen so that $\left|\theta_{d}<\frac{\pi}{2}\right|$ in the next section. From (6) into (3) the closed loop system for the $z$ dynamics is

$$
\ddot{z}+2 \dot{z}+\left(z-z_{d}\right)=\frac{\epsilon_{2}}{m}
$$

then $z \rightarrow z_{d}, \dot{z} \rightarrow 0$ and $u \rightarrow \frac{m g}{\cos \theta_{d}}$. Notice that the convergence rate is exponential.

From the above it follows that for a time arbitrarily long the $x$ dynamics reduces to

$$
\frac{\ddot{x}}{g}=\tan \theta_{d}
$$




\section{Horizontal displacement control $(x)$}

Consider the following function $\theta_{d}$ which depends on $x$ and $\dot{x}$

$$
\theta_{d}=-k_{1} \tanh \left(\frac{\dot{x}}{g}\right)-k_{2} \tanh \left(\frac{(x+\dot{x})}{g}\right)
$$

where $0<k_{i}<\pi / 4$ for $i=1,2$. The control input $\tau$ will be chosen so that $\theta$ converges exponentially to $\theta_{d}$. The expression for $\tau$ is given in section 6 . Notice from the above that $\left|\theta_{d}<\frac{\pi}{2}\right|$ and this avoids the singularity in (6). Therefore we will assume that after some transient $\theta=\theta_{d}$. The RHS of (8) could then be rewritten as

$$
\tan \theta_{d}=-\tan (A+B)
$$

where

$$
A \triangleq k_{1} \tanh \left(\frac{\dot{x}}{g}\right) ; \quad B \triangleq k_{2} \tanh \left(\frac{(x+\dot{x})}{g}\right)
$$

Consider the following trigonometric identity

$$
\tan (A+B)=\frac{\tan A+\tan B}{D}
$$

where

$$
D \triangleq 1-\tan A \tan B
$$

Notice that $D \neq 0$ since $0<k_{i}<\frac{\pi}{4}$. We define

$$
y \triangleq \frac{x}{g}
$$

(9) can also be rewritten as

$$
\tan \theta_{d}=-\frac{\tan A+\tan B}{D}=-\frac{\sigma_{1}(\dot{y})+\sigma_{2}(y+\dot{y})}{D}
$$

where

$$
\begin{aligned}
\sigma_{1}(\dot{y}) & =\tan \left(k_{1} \tanh \dot{y}\right) \\
\sigma_{2}(y+\dot{y}) & =\tan \left(k_{2} \tanh (y+\dot{y})\right)
\end{aligned}
$$

Then from (8), (14) and (15) it follows

$$
\ddot{y}=-\frac{1}{D}\left[\sigma_{1}(\dot{y})+\sigma_{2}(y+\dot{y})\right]
$$

Next it will be proved that the closed loop system (17) is asymptotically stable using a Lyapunov approach. We propose the following Lyapunov function candidate

$$
V=\int_{0}^{y+\dot{y}} \sigma_{2}(\xi) d \xi+\frac{1}{2} \dot{y}^{2}
$$


Notice that $V$ is positive definite since $x_{i} \sigma\left(x_{i}\right)>0$. Differentiating we obtain:

$$
\begin{aligned}
\dot{V} & =\sigma_{2}(y+\dot{y})[\dot{y}+\ddot{y}]+\dot{y} \ddot{y} \\
& =\sigma_{2}(y+\dot{y}) \dot{y}+\left[\sigma_{2}(y+\dot{y})+\dot{y}\right]\left(-\frac{1}{D}\left[\sigma_{1}(\dot{y})+\sigma_{2}(y+\dot{y})\right]\right) \\
& =\sigma_{2}(y+\dot{y}) \dot{y}-\frac{1}{D}\left[\sigma_{2}(y+\dot{y}) \sigma_{1}(\dot{y})+\sigma_{2}^{2}(y+\dot{y})+\sigma_{1}(\dot{y}) \dot{y}+\sigma_{2}(y+\dot{y}) \dot{y}\right] \\
& =\frac{1}{D} \sigma_{2}(y+\dot{y}) \dot{y}\left[1-\sigma_{1}(\dot{y}) \sigma_{2}(y+\dot{y})\right]-\frac{1}{D}\left[\sigma_{2}(y+\dot{y}) \sigma_{1}(\dot{y})+\sigma_{2}^{2}(y+\dot{y})+\sigma_{1}(\dot{y}) \dot{y}+\sigma_{2}(y+\dot{y}) \dot{y}\right] \\
& =-\frac{1}{D}\left[\sigma_{2}^{2}(y+\dot{y}) \sigma_{1}(\dot{y}) \dot{y}+\sigma_{1}(\dot{y}) \sigma_{2}(y+\dot{y})+\sigma_{2}^{2}(y+\dot{y})+\sigma_{1}(\dot{y}) \dot{y}\right] \\
& \leq-\frac{1}{D}\left[\sigma_{2}^{2}(y+\dot{y}) \sigma_{1}(\dot{y}) \dot{y}-\frac{1}{2} \sigma_{1}^{2}(\dot{y})-\frac{1}{2} \sigma_{2}^{2}(y+\dot{y})+\sigma_{2}^{2}(y+\dot{y})+\sigma_{1}(\dot{y}) \dot{y}\right] \\
& \leq-\frac{1}{D}\left[\sigma_{2}^{2}(y+\dot{y}) \sigma_{1}(\dot{y}) \dot{y}+\frac{1}{2} \sigma_{2}^{2}(y+\dot{y})+\frac{1}{2} \sigma_{1}(\dot{y}) \dot{y}\right] \\
& <0
\end{aligned}
$$

where we have used the facts that

$$
-\sigma_{1} \sigma_{2} \leq \frac{1}{2} \sigma_{1}^{2}+\frac{1}{2} \sigma_{2}^{2}
$$

and

$$
\sigma_{1}^{2}(\dot{y}) \leq \dot{y} \sigma_{1}(\dot{y})
$$

or

$$
\left|\sigma_{1}(\dot{y})\right| \leq|\dot{y}|
$$

which is equivalent to:

$$
\begin{array}{lll}
\dot{y}-\sigma_{1}(\dot{y})>0 & \text { for } \quad \dot{y}>0 \\
\dot{y}-\sigma_{1}(\dot{y})<0 & \text { for } \quad \dot{y}<0
\end{array}
$$

Since $\sigma_{1}(\dot{y})$ is a saturation function (see $(16)$ ) it is clear that the above inequalities hold for large values of $\dot{y}$ or $-\dot{y}$. Figure 2 shows that the inequalities also holds for values around the origin for the upper-bound of $k_{1}=\frac{\pi}{4}$.

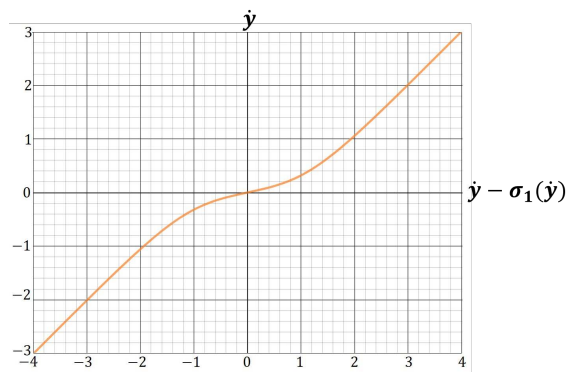

Figure 2: Plot of $\dot{y}-\sigma_{1}(\dot{y})$ showing that inequalities 22 hold.

We conclude $V$ is positive definite and $\dot{V}$ is negative definite. Therefore $y$ and $\dot{y}$ converge to zero, i.e. $x$ and $\dot{x}$ converge to zero. 


\section{Attitude control $(\theta)$}

The torque control input $\tau$ is given by

$$
\tau=\ddot{\theta}_{d}-2\left(\dot{\theta}-\dot{\theta}_{d}\right)-\left(\theta-\theta_{d}\right)
$$

where

$$
\begin{aligned}
& \theta_{d}=-k_{1}\left[\tanh \left(\frac{\dot{x}}{g}\right)\right]-k_{2}\left[\tanh \left(\frac{x+\dot{x}}{g}\right)\right] \\
& \dot{\theta}_{d}=-k_{1}\left[\left(\frac{\ddot{x}}{g}\right) \operatorname{sech}^{2}\left(\frac{\dot{x}}{g}\right)\right]-k_{2}\left[\left(\frac{\ddot{x}+\dot{x}}{g}\right) \operatorname{sech}^{2}\left(\frac{\dot{x}+x}{g}\right)\right] \\
& \ddot{\theta}_{d}=2 k_{1}\left[\left(\frac{\ddot{x}}{g}\right)^{2} \tanh \left(\frac{\dot{x}}{g}\right) \operatorname{sech}^{2}\left(\frac{\dot{x}}{g}\right)\right]+2 k_{2}\left[\left(\frac{\ddot{x}+\dot{x}}{g}\right)^{2} \tanh \left(\frac{\dot{x}+x}{g}\right) \operatorname{sech}^{2}\left(\frac{\dot{x}+x}{g}\right)\right]- \\
& -k_{1}\left[\left(\frac{\dddot{x}}{g}\right) \operatorname{sech}^{2}\left(\frac{\dot{x}}{g}\right)\right]-k_{2}\left[\left(\frac{\dddot{x}+\ddot{x}}{g}\right) \operatorname{sech}^{2}\left(\frac{\dot{x}+x}{g}\right)\right]
\end{aligned}
$$

where $\ddot{x}$ and $\dddot{x}$ are not required to be measured since they are given by the expressions (see (2) and (6))

$$
\begin{aligned}
\ddot{x} & =u \sin \theta_{d} \\
\dddot{x} & =\dot{u} \sin \theta_{d}+u \dot{\theta_{d}} \cos \theta_{d} \\
u & =\frac{m}{\cos \theta_{d}}\left[g-2 \dot{z}-\left(z+z_{d}\right)\right] \\
\dot{u} & =\frac{m}{\cos \theta_{d}}[-2 \ddot{z}-\dot{z}]-\frac{m\left[g-2 \dot{z}-\left(z+z_{d}\right)\right]\left(-\dot{\theta}_{d} \sin \theta_{d}\right)}{\cos ^{2} \theta_{d}}
\end{aligned}
$$

where $\ddot{z}$ can be computed from (7) in terms of $z$ and $\dot{z}$. Substituting (23) into (1) it follows

$$
\ddot{\theta}-\ddot{\theta}_{d}+2\left(\dot{\theta}-\dot{\theta}_{d}\right)+\left(\theta-\theta_{d}\right)=0
$$

Therefore $\theta-\theta_{d}$ and $\dot{\theta}-\dot{\theta}_{d}$ converge to zero.

The control input (6) is basically composed of a nonlinear compensation of $\cos \theta_{d}$, and a linear state feedback. If the control input $u$ has to satisfy a lowerbound $(u>0)$ and an upperbound, we can replace the state feedback part on $z$ and $\dot{z}$ by saturation functions as in (16) such that the closed loop for the $z$ dynamics will have the form given in (17) with $D=1$. The corresponding values of $k_{1}$ and $k_{2}$ could then be used to reduce the size of the saturation functions in $(16)$ as well as to reduce the upperbound of $\left|\theta_{d}\right|$ in $(9)$.

\section{Simulation results}

This section presents the numerical simulation results obtained with the proposed controller. Furthermore we will compare this algorithm with respect to another control algorithm. The second control algorithm which we will call State Feedback is obtained as follows. The altitude $z$ will be controlled as before by using the control input $u$ 
in (6). The torque control input $\tau$ will be given also as before, i.e. as in (23) but the desired angular position $\theta_{d}$ will be simplified as follows

$$
\theta_{d}=-k_{1}\left(\frac{\dot{x}}{g}\right)-k_{2}\left(\frac{x+\dot{x}}{g}\right)
$$

Therefore the State Feedback control algorithm is similar to the proposed algorithm but with a desired angle $\theta_{d}$ which is linear with respect to $x$ and $\dot{x}$. The stability of this State Feedback control strategy is obtained from (7) for the altitude dynamics $z$, from (23) for the convergence of $\theta$ to $\theta_{d}$, and from (8) and (27) for the local stability of the $x$ dynamics assuming that $\theta$ is so small that $\tan (\theta) \approx \theta$. We may expect a similar behavior of the two control algorithms when $x$ and $\dot{x}$ are close to the origin.

In the simulations we have used the following controller parameters for both algorithms: $m=1, k_{a}=20, k_{b}=10, k_{1}=5$ and $k_{2}=3$. We have used the following initial conditions for $z$ and $\theta: z(0)=0.5$ and $\theta(0)=0$. The desired altitude is $z_{d}=1$. We have run 2 simulations with 2 different initial conditions for $x$. Figures 3 and 4 present the results for $x(0)=0.5$. Figures 5 and 6 present the results for $x(0)=50$. It can be seen during the transient the torque control input $\tau$ is larger in the case of the State Feedback control law.

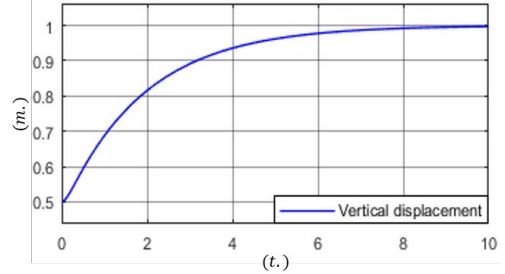

$((\mathrm{a})) z$

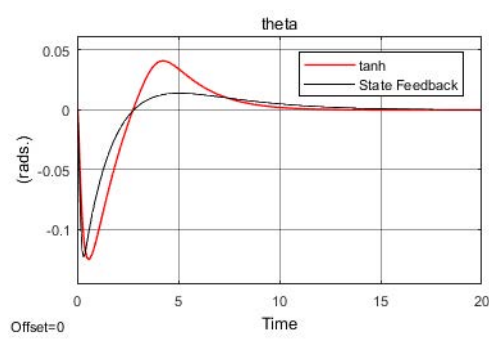

((b)) $\theta$

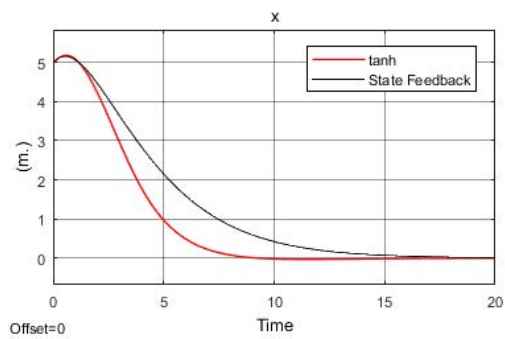

((c)) $x$

Figure 3: The vertical displacement $z$, the angular displacement $\theta$ and the horizontal displacement $x$ with initial condition $x(0)=0.5$

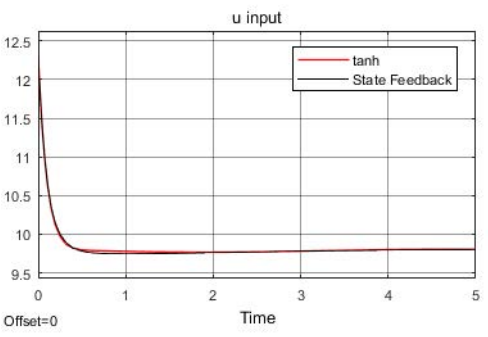

$((\mathrm{a})) u$

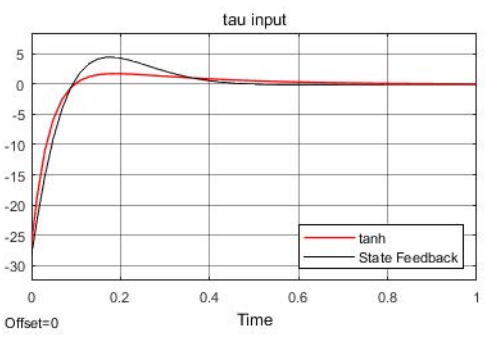

((b)) $\tau$

Figure 4: Control inputs $u$ and $\tau$ with initial condition $x(0)=0.5$ 


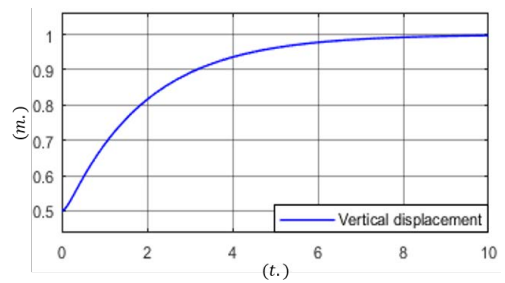

$((\mathrm{a})) z$

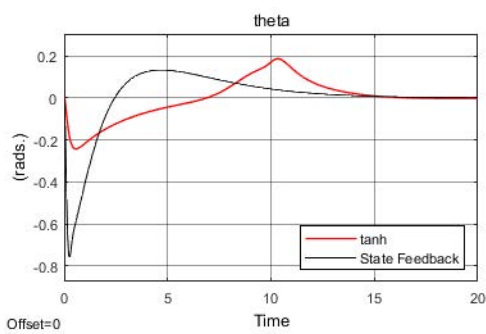

((b) $\theta$

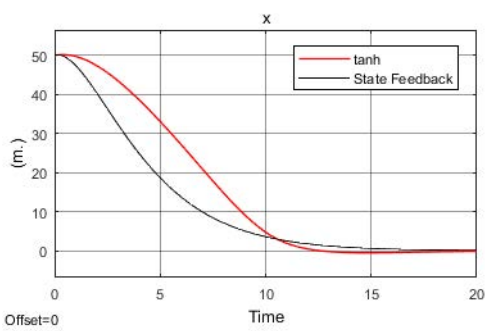

((c)) $x$

Figure 5: The vertical displacement $z$, the angular displacement $\theta$ and the horizontal displacement $x$ with initial condition $x(0)=50$

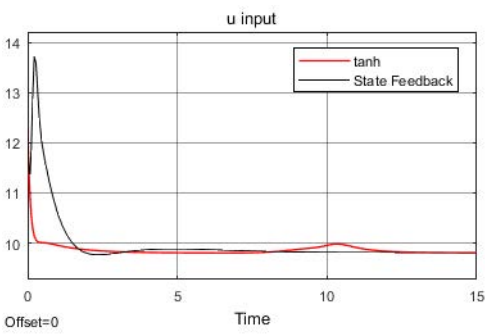

((a)) $u$

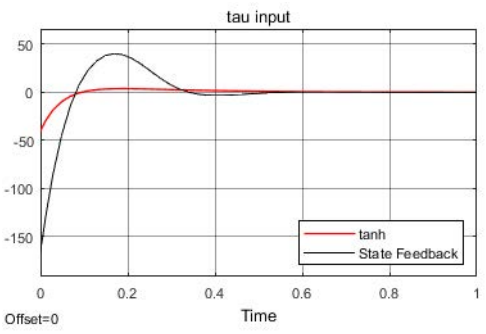

((b)) $\tau$

Figure 6: Control inputs $u$ and $\tau$ with initial condition $x(0)=50$

\section{Acknowledgments}

This project was funded by UTC, CNRS, UMR 7253 Heudiasyc and UMI-LAFMIA 3175 CNRS, CINVESTAV. The authors are grateful to CONACYT (National Council of Science and Technology) for its support.

\section{Data Availability Statement}

The data that support the findings of this study are openly available in https://figshare.com at http://doi.org/10.6084/m9.figshare.16599044.

\section{Conclusions}

This paper has presented a new control strategy for the well known problem of the PVTOL. The altitude control has been obtained by non linear compensation which guarantees that the altitude will reach the desired altitude in a short period of time. A desired orientation has been proposed as a function of smooth saturation function of $x$ and $\dot{x}$. It has been proved that when the orientation reaches the desired orientation then the closed loop for the horizontal displacement is Lyapunov stable. The proposed control strategy has been tested in numerical simulations. 


\section{References}

[1] Hauser J, Sastry S, Meyer G. Nonlinear control design for slightly non-minimum phase systems: Application to V/STOL aircraft. Automatica 1992; 28(4), 665-679. https://doi.org/10.1016/0005-1098(92)90029-F

[2] Lin F, Zhang W, Brandt RD. Robust hovering control of a PVTOL aircraft. IEEE Transactions on Control Systems Technology 1999, 7(3);343-351.10.1109/87.761054

[3] Jadbabaie A, Hauser J. Control of a thrust-vectored flying wing: a receding horizon-LPV approach. International Journal of Robust and Nonlinear Control: IFACAffiliated Journal 2002, 12(9), 869-896. https://doi.org/10.1002/rnc.708

[4] Xin M, Pan H. Robust control of PVTOL aircraft with a nonlinear optimal control solution. Journal of Aerospace Engineering 2010; 23(4), 265-275. https://doi.org/10.1061/(ASCE)AS.1943-5525.0000039

[5] Raffo GV, Ortega MG, Rubio FR. Robust nonlinear control for path tracking of a quad-rotor helicopter. Asian Journal of Control 2015; 17(1), 142-156. https://doi.org/10.1002/asjc.823

[6] Olfati-Saber R. Global configuration stabilization for the VTOL aircraft with strong input coupling. IEEE transactions on Automatic Control 2002; 47(11), 1949-1952. 10.1109/TAC.2002.804457

[7] Huang CS, Yuan K. Output tracking of a non-linear non-minimum phase PVTOL aircraft based on non-linear state feedback control. International Journal of Control 2002, 75(6), 466-473. https://doi.org/10.1080/00207170210121907

[8] Do KD, Jiang ZP, Pan J. On global tracking control of a VTOL aircraft without velocity measurements. IEEE Transactions on Automatic Control 2003; 48(12), 2212-2217. 10.1109/TAC.2003.820148

[9] Shu Z, Lam J. An augmented system approach to static output-feedback stabilization with performance for continuous-time plants. International Journal of Robust and Nonlinear Control: IFAC-Affiliated Journal 2009; 19(7), 768-785. https://doi.org/10.1002/rnc.1348

[10] Consolini L, Tosques M. A homotopy method for exact output tracking of some non-minimum phase nonlinear control systems. International Journal of Robust and Nonlinear Control: IFAC-Affiliated Journal 2009; 19(10), 1168-1196. https://doi.org/10.1002/rnc.1378

[11] Zhu B, Wang X, Cai KY. Approximate trajectory tracking of input-disturbed PVTOL aircraft with delayed attitude measurements. International Journal of Robust and Nonlinear Control 2010; 20(14), 1610-1621. https://doi.org/10.1002/rnc.1536

[12] Marconi L, Naldi R, Isidori A. High-gain output feedback for a miniature UAV. International Journal of Robust and Nonlinear Control 2014; 24(6), 1104-1126. https://doi.org/10.1002/rnc.2959

[13] Aguilar-Ibanez C, Sira-Ramirez H, Suarez-Castanon MS, Garrido R. Robust trajectory-tracking control of a PVTOL under crosswind. Asian Journal of Control 2019; 21(3), 1293-1306. https://doi.org/10.1002/asjc.1817 
[14] Zhao B, Xian B, Zhang Y, Zhang X. Nonlinear robust sliding mode control of a quadrotor unmanned aerial vehicle based on immersion and invariance method. International Journal of Robust and Nonlinear Control 2015; 25(18), 3714-3731. https://doi.org/10.1002/rnc.3290

[15] Chen F, Jiang R, Zhang K, Jiang B, Tao G. Robust backstepping sliding-mode control and observer-based fault estimation for a quadrotor UAV. IEEE Transactions on Industrial Electronics 2016; 63(8), 5044-5056. 10.1109/TIE.2016.2552151

[16] Yu-Chan C, Bao-Li M, Wen-Jing X. Robust stabilization of nonlinear PVTOL aircraft with parameter uncertainties. Asian Journal of Control 2017; 19(3), 12391249. https://doi.org/10.1002/asjc.1411

[17] Aguilar-Ibanez C. Stabilization of the PVTOL aircraft based on a sliding mode and a saturation function. International Journal of Robust and Nonlinear Control 2017; 27(5), 843-859. https://doi.org/10.1002/rnc.3601

[18] Abdessameud A, Tayebi A. Global trajectory tracking control of VTOLUAVs without linear velocity measurements. Automatica 2010; 46(6), 1053-1059. https://doi.org/10.1016/j.automatica.2010.03.010

[19] Su S, Lin Y. Robust output tracking control for a velocity-sensorless vertical take-off and landing aircraft with input disturbances and unmatched uncertainties. International Journal of Robust and Nonlinear Control 2013; 23(11), 1198-1213. https://doi.org/10.1002/rnc.2813

[20] Aguilar-Ibáñez C, Sossa-Azuela JH, Suarez-Castanon MS. A backstepping-based procedure with saturation functions to control the PVTOL system. Nonlinear Dynamics 2016; 83(3), 1247-1257. https://doi.org/10.1007/s11071-015-2400-y

[21] Jia Z, Yu J, Mei Y, Chen Y, Shen Y, Ai X. Integral backstepping sliding mode control for quadrotor helicopter under external uncertain disturbances. Aerospace Science and Technology 2017; 68, 299-307. https://doi.org/10.1016/j.ast.2017.05.022

[22] Chemori A, Marchand N. A prediction-based nonlinear controller for stabilization of a non-minimum phase PVTOL aircraft. International Journal of Robust and Nonlinear Control: IFAC-Affiliated Journal 2008; 18(8), 876-889. https://doi.org/10.1002/rnc.1248

[23] Wu SL, Chen PC, Hsu CH, Chang KY. Gain-scheduled control of PVTOL aircraft dynamics with parameter-dependent disturbance. Journal of the Franklin Institute 2008; 345(8), 906-925. https://doi.org/10.1016/j.jfranklin.2008.05.006

[24] Esteban S, Gordillo F, Aracil J. Three-time scale singular perturbation control and stability analysis for an autonomous helicopter on a platform. International Journal of Robust and Nonlinear Control 2013; 23(12), 1360-1392. https://doi.org/10.1002/rnc.2823

[25] Chadli M, Aouaouda S, Karimi HR, Shi P. Robust fault tolerant tracking controller design for a VTOL aircraft. Journal of the Franklin Institute 2013; 350(9), 2627-2645. https://doi.org/10.1016/j.jfranklin.2012.09.010

[26] Brandão AS, Gandolfo D, Sarcinelli-Filho M, Carelli R. PVTOL maneuvers guided by a high-level nonlinear controller applied to a rotorcraft machine. European Journal of Control 2014; 20(4), 172-179. https://doi.org/10.1016/j.ejcon.2014.04.003 
[27] Kuga S, Nakamura H, Satoh Y. Robust Adaptive Control of PVTOL System via Minimum Projection Method. Journal of Mathematics and System Science 6 2016; 169-179. 10.17265/2159-5291/2016.05.001

[28] Tayebi A, McGilvray S. Attitude stabilization of a VTOL quadrotor aircraft. IEEE Transactions on control systems technology 2006; 14(3), 562-571. 10.1109/TCST.2006.872519

[29] Benrezki RR, Tayebi A, Tadjine M. Adaptive trajectory tracking control for VTOL-UAVs with unknown inertia, gyro-bias, and actuator LOE. International Journal of Robust and Nonlinear Control 2018; 28(17), 5247-5261. https://doi.org/10.1002/rnc.4308

[30] Zou Y. Nonlinear robust adaptive hierarchical sliding mode control approach for quadrotors. International Journal of Robust and Nonlinear Control 2017; 27(6), 925-941. https://doi.org/10.1002/rnc.3607

[31] Sanahuja G, Castillo P, Sanchez. A Stabilization of $n$ integrators in cascade with bounded input with experimental application to a VTOL laboratory system. International Journal of Robust and Nonlinear Control: IFAC-Affiliated Journal 2010; 20(10), 1129-1139. https://doi.org/10.1002/rnc.1494

[32] Castillo P, Dzul A, Lozano R. Real-time stabilization and tracking of a four-rotor mini rotorcraft. IEEE Transactions on control systems technology 2004, 12(4), 510516. 10.1109/TCST.2004.825052

[33] Teel AR. Global stabilization and restricted tracking for multiple integrators with bounded controls. Systems \& control letters 1992; 18(3), 165-171. https://doi.org/10.1016/0167-6911(92)90001-9

[34] Tran TT, Ge SS, He W. Adaptive control of a quadrotor aerial vehicle with input constraints and uncertain parameters. International Journal of Control 2018; 91(5), 1140-1160. https://doi.org/10.1080/00207179.2017.1309572

[35] Aguilar-Ibañez C. Stabilization of the PVTOL aircraft based on a sliding mode and a saturation function. International Journal of Robust and Nonlinear Control 2017, 27(5), 843-859. https://doi.org/10.1002/rnc.3601

[36] Naldi R, Furci M, Sanfelice RG, Marconi L. Robust global trajectory tracking for underactuated VTOL aerial vehicles using inner-outer loop control paradigms. IEEE Transactions on Automatic Control 2016; 62(1), 97-112. 10.1109/TAC.2016.2557967 